\title{
Letteratura dialettale come caratteristica della «letteratura minoritaria»
}

\author{
Jadranka Cergol
}

Lo scopo dell'articolo è di provare che l'espressione artistica dialettale è una caratteristica tipica della letteratura minoritaria. Le ricerche hanno infatti finora dimostrato che gli autori che decidono di esprimersi originalmente nella forma dialettale, provengono principalmente dalle zone limitrofe di una cultura, nelle quali la lingua entra in contatto con altre realtà linguistiche e culturali.

Parole chiave: letteratura minoritaria, letteratura dialettale, Sloveni in Italia, Italiani in Slovenia e in Croazia, rapporto con la lingua

Namen prispevka je dokazati, da je lahko narečna književnost ena izmed tipičnih značilnosti manjšinske literature. Dosedanje raziskave so namreč pokazale, da izhajajo avtorji, ki se odločajo za izvirno pisanje v narečju, večinoma iz obmejnih območij neke kulture, na katerih prihaja materni jezik v stik z drugimi jezikovnimi in kulturnimi skupnostmi.

Ključne besede: manjšinska književnost, narečna književnost, Slovenci v Italiji, Italijani v Sloveniji in na Hrvaškem, odnos do jezika

T 1 dialetto è probabilmente l'espressione artistica più intima, con la quale gli autori vogliono penetrare nella sfera più profonda del rapporto verso se stessi e il mondo che li circonda. La letteratura dialettale, soprattutto quella slovena, è stata per lunghi anni ignorata dalla critica letteraria per vari motivi: la sua comprensione richiede uno sforzo particolare nel capire il codice linguistico usato, oltre a ciò è necessaria un'approfondita conoscenza culturale, etnica, antropologica e geografica degli autori. Ma probabilmente la ragione più profonda per la quale la poesia dialettale è rimasta trascurata dalla critica letteraria è legata sia allo status sociale più basso degli autori che ad una loro provenienza geografica più periferica rispetto ai centri culturali, come viene ribadito dalla ricercatrice slovena Novak Popov. ${ }^{\mathrm{I}}$ Il fenome-

Irena Novak Popov, "Razmišljanje o sodobni narečni poeziji," in Slovenska narečja med sistemom in rabo, ur. Vera Smole (Ljubljana: Znanstvena založba Filozofske, 2009), 435 . no ha avuto un riscontro diverso nella letteratura italiana, per la quale il dialetto è parte integrante ed ineliminabile. ${ }^{2}$ Anche in Italia però la poesia dialettale ha vissuto un momento di crisi in concomitanza con i grandi cambiamenti sociali del dopoguerra che hanno portato ad una maggiore alfabetizzazione della popolazione e al lento ma inesorabile abbandono del dialetto rispetto alla lingua standard. Negli ultimi decenni del Novecento però la letteratura dialettale e soprattutto la poesia dialettale vive un periodo di rinascita anche in Italia. ${ }^{3}$ Così come in Italia questa tipologia di espressione artistica sta prendendo in $\mathrm{Eu}-$ ropa un particolare valore, non solo letterario, ma anche antropologico ed etnico: la forma dia-

2 Gianfranco Contini, La letteratura italiana (Firenze-Milano: Sanoni-Accademia, 1974), 45 .

3 Luigi M. Cesaretti Salvi. "Le letteratura dialettale." Enciclopedia Treccani (2006), http://www.treccani.it/enciclopedia/letteratura-dialettale_\%28Enciclopedia-Italiana\%29/(28-4-2017) 
lettale sta diventando la nuova forma di espressione di quelle radici culturali, senza le quali le varie culture non riuscirebbero a trarre linfa vitale per la loro esistenza futura. La letteratura dialettale, e in particolare la sua espressione poetica, si sta quindi presentando come un ramo che cresce rigoglioso parallelamente alla letteratura scritta nella lingua standard. Di questo si rendono conto soprattutto i poeti e le poetesse dialettali che controtendenza e contro la convenzione decidono di usare la loro parlata locale anche per narrare le loro storie o esprimere se stessi in versi. Questo fatto è particolarmente riscontrabile proprio in modo alquanto evidente nelle letterature minoritarie, sia all'interno del sistema letterario degli Italiani in Slovenia e in Croazia, come è stato già dimostrato sia nell'antologia di Nelida Milani e Roberto Dobran ${ }^{4}$ che in quella della prof. Zudič Antonič, 5 ma anche quasi contemporaneamente anche nel sistema letterario degli Sloveni in Italia, come viene invece dimostrato dai ricercatori Miran Kosuta, David Bandelj e la sottoscritta. Infine, nel 2007 è uscita una consistente monografia scientifica della ricercatrice slovena Marija Stanonik ${ }^{6}$ che rappresenta un'analisi completa e approfondita di tutta la letteratura dialettale slovena.

Come viene definita da Miran Hladnik, la letteratura dialettale è una "forma di letteratura scritta in un dialetto di una qualsiasi lingua letteraria. Secondo i criteri più ristrettivi, rientrano in essa soltanto quelle opere che sono nate dopo la standardizzazione della lingua, secondo invece i limiti più ampi, possono rientrare anche le opere delle letteratura orale ovvero le opere folkloristiche dai tempi prima della standardizzazione della lingua." La critica letteraria di entrambe le lingue di norma non prende in considerazione le opere popolari e folkloristiche che

$4 \quad$ Nelida Milani and Roberto Dobran, Le parole rimaste (Pula: Pietas Iulia; Rijeka: Edit, 2010), 5 I e seg.

5 Nives Zudič Antonič, Storia e antologia della letteratura italiana di Capodistria, Isola e Pirano (Capodistria: Unione italiana, 2014).

6 Marija Stanonik, Slovenska narećna književnost (Maribor: Slavistično društvo Maribor, 2007)

7 Miran Hladnik, "Regionalism in Slovene Rural Prose," Slovene Studies 13, no.2 (1991): 143-53. sono nate sì in dialetto, ma perché non c'era la possibilità di usare nessun altro codice linguistico. Mi sembra quindi opportuno distinguere tra coloro che coscientemente decidono di usare il dialetto al posto della lingua standard e coloro che invece usavano il dialetto perché non conoscevano altra forma espressiva. Soltanto nel momento in cui si arriva nella società ad un avanzato processo di scolarizzazione della popolazione che usa il linguaggio standard e si ha quindi la possibilità di scegliere coscientemente se usare la lingua standard o la parlata locale, allora è possibile parlare anche di una letteratura dialettale riflessa, cioè tale, nella quale gli autori scelgono il dialetto con un determinato scopo molto preciso, e cioè quello di dare precedenza al dialetto. Ed è per questa ragione che nell'articolo non verranno presi in considerazione autori dei secoli passati come neanche non verrà presa in considerazione la letteratura folkloristica che ha altri tipi di caratteristiche e deve essere analizzata con altri parametri letterari.

\section{Letteratura minoritaria}

Sulla letteratura minoritaria di entrambe le comunità linguistiche sono state già svolte delle ricerche che sono sfociate per la parte italiana nei due volumi della Milani e di Dobran e adesso recentemente anche nella monografia della prof. Nives Zudič Antonič; per la parte slovena invece vanno citate le antologie di David Bandelj per la poesia e le monografie scientifiche di Miran Košuta e di Marija Pirjevec. ${ }^{8}$ Inoltre nel 2006 sono uscite due antologie bilingui: Dru gačni verzi: pesniki dveh manjšin / Versi diversi: poeti di due minoranze curate da Miran Košuta per la parte slovena e da Elis Deghenghi Olujić per quella italiana che raccolgono e mettono a confronto i poeti delle due minoranze. Anche la sottoscritta aveva già pubblicato un articolo sulle caratteristiche principali della letteratura mi-

8 Miran Košuta, E-maili: eseji o mejni literaturi (Maribor: Litera, 2008); David Bandelj, Rod Lepe Vide: antologija sodobne poezije Slovencev v Italiji (Ljubljana: Študentske založba, 2009); David Bandelj "Literature of Slovenians in Italy: A subsystem of Slovenian supernational system?" Interlitteraria 5, no.2 (2010): 432-44I; Marija Pirjevec, Questa Trieste (Trieste: Mladika, 2016) 
noritaria: ${ }^{9}$ è stato dimostrato che la letteratura minoritaria è caratterizzata da una dimensione ontologica, da un'innata interculturalità, dalla stigmatizzazione nazionale, da un innato attaccamento alla regione e al territorio d'appartenenza, dal valore linguistico e dalla memoria storica. Il presente articolo ha lo scopo di analizzare soltanto la dimensione linguistica e in specifico l'espressione dialettale.

\section{La dimensione linguistica}

Tra tutte le dimensioni sopra elencate rappresenta la lingua dell'autore quel valore che è strettamente connesso alla stigmatizzazione nazionale, come portatore principale della dimensione ontologica. Studiando il rapporto degli autori minoritari nei confronti della loro lingua madre si può riscontrare una particolare relazione che comprende anche un senso di difficoltà a padroneggiare la propria lingua madre che bisogna impararla, svilupparla e mantenerla viva in un contesto sociale nel quale questa stessa lingua non viene usata.

\section{La maestra con la macchina rossa e gli occhi neri dice che scrivo male in italiano. Fa che diventi bravo e che agli esami, quando la ma- estra farà il dettato, scriva in modo corretto tutte quelle parole che hanno le doppie. Fa che la signora Slapnik, dalla quale mi manda la mamma, mi insegni, come si parla lo slove- nobene e in modo corretto. ${ }^{\text {Io }}$}

Si tratta di quella mancanza di disinvoltura o scioltezza, della quale scrive Miran Kosuta, che può portare addirittura ad un eccessiva accuratezza della lingua, perché la parola slovena significa anche la lotta per la propria identità minacciata e per la propria libertà linguistica. ${ }^{\mathrm{II}}$ Per entrambe le minoranze linguistiche è caratteristico che sul piano linguistico evitino le forme

9 Jadranka Cergol, "Some Typological Features of,Minority' Literatures: the Case of the Slovenian and Italian Minorities." L'analisi linguistica eletteraria 24, no. I (2016): 61-76.

Io Marko Sosič, Tito, amor mijo (Trieste: Comunicarte, 2012), 13.

I Košuta, E-maili: eseji o mejni literaturi; Marija Pirjevec, $»$ Periodizacija slovenske književnosti na Tržaškem (od 16. do 20. stoletja) «, Annales, Series historia et sociologia 21, no.2 (2011): 360. estreme di sperimentalismo linguistico, il ludismo, il dadaismo ecc., ma si è alla continua "ricerca della letterarietà, in un certo senso un lavoro teorico e poetico sulla scrittura; quindi in fondo, a volte tra le righe e a volte apertamente, è all'opera una forte attenzione alla componente estetica".

Il rapporto nei confronti della lingua madre e/o del dialetto locale è espresso molto chiaramente anche dal seguente passo, tratto dal romanzo Martin Muma di Ligio Zanin, pure lui poeta dialettale.

Era un appassionato della felicissima teoria, specialmente per Martino, dei linguaggi regionali italiani. Secondo la quale, in breve, la lingua italiana pura sarebbe un desiderio, un sogno, a cui si avvicinerebbero pochi: eminenti linguisti, alcuni giornalisti dell'EIAR e diversi italianisti. Mentre la maggioranza, stragrande, anche delle persone dotte, parlerebbe e scriverebbe una lingua, colorita e profumata variamente, in conformità del particolare humus linguistico, i dialetti, delle regioni d'Italia, sempre nel rispetto delle regole fondamentali dell'italiano. Il professor Callegarini, perciò raccomandò a mamma Checchina, sin dal primo colloquio, di parlare al figlio come meglio sapeva, cioè in rovignese. Dialetto nobile che traeva le sue origini direttamente dal parlato latino, ricco di simboli, di immagini, di modi di dire, fioriti, attraverso quasi due millenni, nel quotidiano di quella gente italiana. Il figlio, poi, studiando, sarebbe stato in grado di porgere quegli antichi doni al dialetto della propria nazione, e, attraverso questa, a tutte le altre. [...]

E Martino, prossimo a finire le magistrali, a maledir la cattiva sorte che gli aveva dato in tutti quegli anni quella bonadagninte, quella buon a nulla, che lo aveva sempre bloccato con il suo spappagallare il fiorentino. Ora con Callegarini, che sapeva favalà, cioè parlare, essendo un uomo e non un vaso di pitI2 Milani, Dobran: Le parole rimaste II, 338 . 
tura, si esprimeva senza paura, a non finire.

Non aveva più paura, chè, se i pescatori di Aci Trezza parlavano per la penna di Verga, consigliatogli dal professore, un italiano dal profumo siciliano, anche lui poteva dir qualcosa che avesse l'aroma del ginepro istriano. L'Istria non aveva nulla da vergognarsi al cospetto della Sicilia. ${ }^{13}$

\section{Letteratura minoritaria dialettale}

Da una veloce analisi delle varie letterature europee emerge che non tutte le nazioni hanno una propria letteratura dialettale che molto spesso nasce a causa della "mancanza di una politica centralizzante dello stato nazionale, di una mancanza di un unico centro culturale, del frazionamento amministrativo ovvero di una tradizione culturale regionale". ${ }^{14}$ La letteratura dialettale è così molto sviluppata in Croazia, in Italia, in Slovenia, in Austria, in Germania e in America, mentre non la conoscono gli Inglesi, i Francesi e i Serbi. ${ }^{15}$ Dalla monografia scientifica della prof. Marija Stanonik Slovenska narečna književnost ${ }^{16}$ emerge che la letteratura dialettale è nata prima di tutto sui confini del territorio etnico sloveno, in ambienti, nei quali vivevano e vivono ancora le minoranza slovene: la Carinzia austriaca, la regione in Ungheria lungo il confine con la Slovenia, e la zona confinaria tra Tarvisio e Muggia in Italia. Soltanto negli ultimi decenni il fenomeno si è allargato nelle restanti parti della Slovenia. Un fenomeno simile è riscontrabile anche nella letteratura della comunità italiana in Slovenia e in Croazia: la produzione letteraria in dialetto è estremamente ricca, la maggior parte degli autori minoritari pubblica opere originali, descrivendo fatti, persone, luoghi, territori, sensazioni che hanno loro stessi vissuto. Il mondo raccontato dai poeti istro-dalmati italiani ci mostra soprattutto un dualismo tra la città e la campagna,

I3 Ligio Zanini, Martin Muma (Fiume: Edit, 1999): I40-I.

I4 Hladnik, "Regionalism in Slovene Rural Prose", I48.

is Hladnik, "Regionalism in Slovene Rural Prose", i 47; Irena Novak Popov, "Razmišljanje o sodobni narečni poeziji," in Slovenska narećja med sistemom in rabo, ur. Vera Smole (Ljubljana: Znanstvena založba Filozofske fakultete, 2009), 435-453

I6 Stanonik, Slovenska narećna književnost, 23-35. si estende alla descrizione del patrimonio culturale dei pescatori e dei contadini, ma è sempre intessuto di una sensazione individuale di se stessi e della comunità, sia della ristretta comunità nazionale italiana, sia della più ampia comunità sociale, tutto ciò espresso nei due dialetti principali, cioè nel dialetto istro-veneto e istro-romanzo.

A riprova del fatto che la letteratura dialettale originale nasce sui confini di un territorio etnico ne è il dato che emerge dall'analisi nella monografia sulla letteratura dialettale slovena, cioè la constatazione che la prima poetessa a imboccare la via dell'espressione dialettale slovena sia stata Marija Mijot, autrice slovena di Trieste che ha pubblicato la sua prima raccolta di poesie in dialetto nel 1962 Souze jn smeh. ${ }^{17}$ Di lei, il suo allora compaesano Vladimir Bartol, scrisse: "Il dialetto non serve all'autrice per descrivere fatti interessanti, lei la lingua dialettale la vive proprio. Anzi, direi che la lingua di Marija Mijot addirittura canta! Il dialetto la ubbidisce e anche se non è particolarmente ricco di significato, lẹi riesce con esso ad esprimere i propri sentimenti più profondi, come vive in esso la propria terra $\mathrm{e}$ la sua gente.... Come un amore sfortunato, semplice, ingenuo, ma comunque genuino". ${ }^{18}$ Sicuramente la poesia della Mijot, intrisa sì di elementi locali, ma legata strettamente alla società culturalmente vivace di Trieste non è fiorita in mezzo ad deserto, se pensiamo al fatto che la Mijot leggeva molto volentieri poeti dialettali come Biagio Marin e sicuramente conosceva i saggi di Pier Paolo Pasolini sul friulano.

Alla stessa maniera anche i critici italiani descrivono la letteratura dialettale italiana in Istria, quando parlano di autori che decidono di usare il dialetto perché questo permette loro una certa verginità, genuinità, rigogliosità rispetto alla lingua standard che loro invece sentono rigida, incolore, vuota, sterile. "Sa [il poeta dialettale], perché non ha mai avuto dubbi, che il dialetto è una lingua vera ed esprime un mondo completo come qualsiasi lingua nazionale, dagli

I7 Marija Mijot, Souze jn smeh (Trst: Založništvo tržaškega tiska, 1962).

i 8 Vladimir Bartol, "Predgovor," in Souze jn smeh, ur. Marija Mijot (Trst: Založništvo tržaškega tiska, 1962), 7 . 
oggetti ai sentimenti: sa che col dialetto può ridere e piangere, narrare aneddoti e parlare di Dio e dell'anima." ${ }^{\text {"9 }}$ Questa affermazione ricorda anche il pensiero della ricercatrice slovena Novak Popov:

Moltidifensori [delle identità regionali] fanno notare anche il rigetto verso l'integralismo nazionale e globale, verso l'uniformazione, verso l'impoverita monoculturalità $\mathrm{e}$ autoritarismo, a causa dei quali vengono in silenzio sacrificati e dimenticati i risultati ottenuti dai gruppi emarginati [...] La poesia dialettale si mostra quindi come un'espressione di libertà, di coraggio, di una forza interiore. ${ }^{20}$

Le tematiche affrontate dai poeti dialettali

Le tematiche affrontate dai poeti dialettali sono di solito di natura molto soggettiva, scrivono prevalentemente di sé, del proprio vissuto quotidiano, dei propri luoghi, del mare e degli ulivi, della vita e dell'amore, dei luoghi che visitano e sui quali riflettono. Come viene però constato dai ricercatori Milani e Dobran, c'è un'altra motivazione, forse addirittura più importante, cioè il livello psicologico della scelta del dialetto che viene definito da Brevini "lingua dell'autobiografia vs lingua dell'anonimato”. ${ }^{21}$ Per gli appartenenti ad una comunità nazionale minoritaria la scelta del dialetto è anche un fattore psicologico di una rivalsa nei confronti della storia che ha visto già molteplici cambiamenti politici: scegliere il dialetto vuol dire quindi ribadire il senso di una diversa appartenenza, di dimostrare delle radici linguistiche e culturali che usano un codice diverso. ${ }^{22}$ Ad esempio il poeta rovignese Giusto Curto descrive così il proprio luogo nella poesia Elmeio logo:

19

Claudio Marabini, "Poesia contemporanea in lingua e dialetto: quale rapporto?" in La maschera del dialetto, ur. Andrea Foschi, Elio Pezzi (Ravenna: Longo, 1988), 64.

20 Novak Popov, "Razmišljanje o sodobni narečni poeziji”, 440.

2 I Franco Brevini, Le parole perdute: Dialetti e poesia del nostro secolo (Torino: Einaudi, 1990): 55

22 Milani, Dobran, Leparole rimaste, 521.
I ma ganbio i drapi e cradime nu mal dol

sa anche i paro oûn pupo da pisiol,

i son sul mieîo e ma sento un gigante

e qua i nu iè pagoûra manco del cavalgante. ${ }^{23}$

Molte delle poesia dialettali ricordano i paesaggi bucolici e idilliaci della vita in campagna ed esprimono spesso il forte attaccamento alla terra sia dell'autore che dell'intera comunità alla quale appartiene. Se la poesia fosse stata scritta nella lingua standard, probabilmente non avremmo potuto captare la genuinità delle sue parole.

Anche la poetessa di Pola Ester Sardoz Barlessi descrive così il profumo dell'Istria:

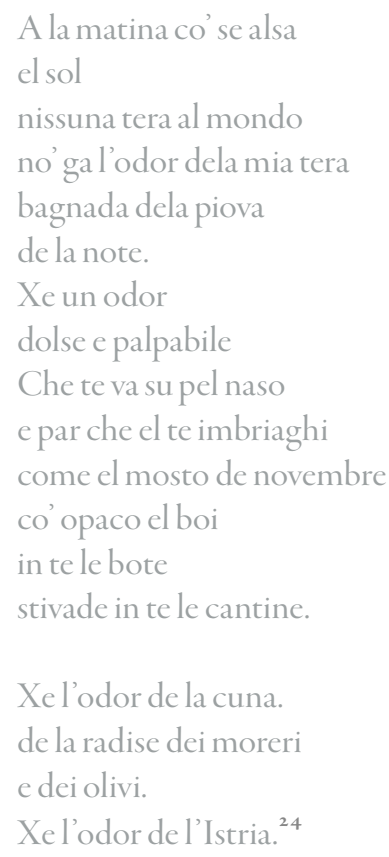

L'attaccamento alla propria terra è un tema prevalente anche nella poesia dialettale slovena in Italia, come lo dimostra la già citata poetessa Marija Mijot di San Giovanni, oggi rione di Trieste, ma cinquant'anni fa ancora villaggio alle porte della città, che descrive così i luoghi della sua infanzia, ai quali sono legati molti ricordi:

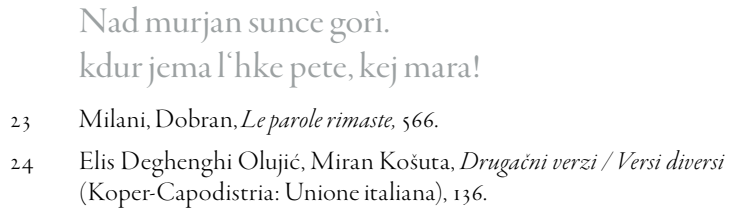


T'n se na s'mnje na Šance vrti,

v's Luonjar, Mandrja, Četnara! $!^{25}$

Ma la descrizione dei luoghi non è l'unico motivo presente nei poeti dialettali. Se decenni fa valeva ancora il sintagma che la poesia dialettale fosse solo una forma di espressione soggettiva, personale, forse una poesia dedicata ai bambini e alla vita campestre, le tematiche degli ultimi anni si sono allargate. Ad esempio il poeta sloveno di Trieste Atilij Kralj scrive prevalentemente poesie umoristiche; in altri poeti è possibile trovare anche il tema del patriottismo, dell'attaccamento alla propria terra natia, alle proprie radici, alla propria storia, ma anche dei sentimenti umani, come l'amore o la sofferenza per la perdita di una persona cara. Se pensiamo a questo fattore sociologico, diventa chiaro che "scrivere nella lingua viva, ovvero nel dialetto, è segno di ribellione di ogni comunità più piccola (anche di una popolazione) nei confronti di una unificazione funzionalista, del mondo globalizzato, dove tutto ciò che è individuale, unico, personale, a misura d'uomo, non vale molto e dovrebbe addirittura scomparire. Molti hanno a questi piccoli gruppi (anche al popolo sloveno) predetto una sorte avversa, ma queste previsioni non si sono avverate, perché in natura ci sono delle forze che remano contro le forze distruttrici, forze che mantengono in vita, vicino alle grandi creature anche creature piccole". ${ }^{26}$ A dimostrazione della tesi di Hočevar citiamo una poesia di Silvana Paletti, poetessa della Resia, dal titolo Ta rozajanski glas, che dimostra l'attaccamento dell'autrice alla parlata locale.

Tu-w ti rožinëj dulïni,
puložanä pod Canïnom,
tej da vilažnji din,
se mi jasnijo lipe biside,
od noga glasa.
Iti, jë glas od me zamje. nnk

25 Marija Mijot, Souze jn smeh (Trieste: Založništvo tržaškega tiska, 1962), 18 .

26 Jože Hočevar, "Pride vse en vse pasá," in Pride vse en vse pasá (Pesmi o Istri in njenih ljudeh), ur. Nelda Štok-Vojska (Marezige: samozaložba, 1999): 148.

\section{Od mlade od särca \\ wzira, pod suncon. \\ Särčne biside mu stjijo. \\ Sam, zna, jübit od rožicöw \\ nu na jasnimö, nëbëske racjune. \\ Iti jë glas, od mëh judi. \\ Iti jë glas, Rozajanski, \\ ki zadavit, ni smin, \\ zabit, ni mörën. \\ Mo mati, za šenk, na mi dala, \\ da ja se znej po svëtö, \\ da ja si maköj Rozajän.}

Iti jë glas, Rozajanski,

ki od vište od sunca,

skryt, ni mörën ...

Zakoj, iti, jë glas

od me duline ..

od me zamje...

od mëh samih judi. ${ }^{27}$

군

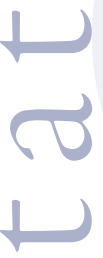

Ciò che abbiamo già notato per Curto, vale anche per la Paletti che si lascia trasportare dalla parlata resiana per descrivere la sua bella valle verde sotto il Kanin, ma che contemporaneamente indirizza il suo pensiero anche verso un altro tema, cioè verso l'uso della lingua, di quella lingua che sua madre le ha donato nella culla e che lei continuerà ad usare, perché è la voce della sua valle, della sua terra e della sua gente.

Sulla lingua e il codice usato riflettono anche altri poeti, tra i quali spicca Libero Benussi che ha descritto la sua parlata nella lirica Ca faviela! (Che favella!): perdere la propria lingua significa per Benussi perdere la propria anima.

\footnotetext{
Nu curo fa altro

ca tirala $\int u$ cul prufoumo del mangrei

e insanbralo cun quil de li greighe

e cun quil dela gruojula.

Nu curo daghe oun culur,

la uo fa quil del marinoun

e quil del $\int$ bulo saltula del sabusein

insanbra cul canto del merlo in amur.

Nu curo darghe armuneia,
}

27 
la uo đa quila de la tiera calda,

quila del busco grungula

e quila del mar ca carisa i denti de can;

\section{sulruso deifeighi. 28}

In entrambi i casi abbiamo potuto notare che le poesie vanno oltre la mera predisposizione individuale, ma si tratta di voci che sono destinate alla comunità più ampia, soprattutto alla comunità linguistica che legge queste poesie e si identifica in esse. Perciò è possibile in alcune di queste poesie ritrovare anche il tema del ricordo storico di una comunità. Un esempio ne è Tino Gavardo, poeta italiano di Capodistria che attacca il potere austro-ungarico, verso il quale non dimostra nessuna predisposizione positiva, a causa della sensazione della perdita dello stato italiano, del rammarico per la caduta della repubblica di Venezia e a causa dell'amarezza per il cambiamento politico della città.

Etuto xe sito; soltanto un momento

se senti una yose che par un lamento,

na vose de pianto che par quasi umana;

xe un boto che manda la vecia campana.

a vecia campana che, a fianco le tori,

ciamava a seduta $\mathrm{i}$ antichi signori,

la vecia campana che dormi lassù,

che sempre ricorda per chi l'à batù... (...)

Ma adesso sti veci che più no comanda,

in morte i patissi l'ofesa più granda;

l'ofesa più granda: cambiarghe la sèna,

menarli in berlina fra i turchi de Viena.

Ma, atenti là in alto che, se anca de gesso,

el cuor de San Marco xe sempre l'istesso;

atenti lá in alto che forsi de novo

no'l vegni rabioso de fora del covo.

Chè alora, se'l urla sto vecio Leon,

al Prater va in tera le quinte e'l tendon...

28

Milani, Dobran, Le parole rimaste I, 612.
O turchi e Viena, porteghe rispeto,

se no, garantisso, fini int-un sguasseto. ${ }^{29}$

\section{Conclusione}

Attraverso gli esempi presentati ho voluto dimostrare che gli appartenenti di due comunità linguistiche minoritarie decidono di usare il dialetto con scopi letterari. La scelta del vernacolo locale non è una scelta frivola, dietro alle confessioni personali possiamo intravedere anche messaggi diretti a tutta la comunità minoritaria e a tutta la società in genere, dato che tangono anche problemi politici, dilemmi linguistici, patrimonio culturale e ricordo storico. Mi è sembrato soprattutto interessante analizzare e dimostrare come queste tematiche che ho appena menzionato sono presenti in due sistemi letterati diversi, che usano due codici linguistici distinti (dialetto sloveno e dialetto istro-veneto), ma che evidentemente vivono la stessa situazione sociale e problemi sia soggettivi che comunitari molto simili. Si tratta quindi di una forma letteraria, quella della letteratura dialettale, che vale la pena ricercare soprattutto per il fatto che sta diventando sempre più consistente e ricca. Anche grazie all'impegno, alla tenacia e al desiderio di coloro che vogliono mantenerla.

\section{Povzetek}

Cilj članka je dokazati, da je narečna literatura ena tipičnih izraznih sredstev manjšinske literature. Pretekle raziskave so dokazale, da različni narečni literarni sistemi so še najprej vzklili na obrobju etničnega naselitvenega prostora, $v$ sosednjih deželah in predvsem $v$ tistih okoljih, v katerih jezik prihaja v stik z drugimi jeziki in kulturami. $\mathrm{Na}$ istih geografskih območjih je odnos do narečja veliko bolj izrazit in pomemben. $Z$ analizo dveh manjšinskih skupnosti, slovenske v Italiji in italijanske v Sloveniji in na Hrvaškem, avtorica poskuša dokazati, da je narečna literatura zelo tipičen fenomen manjšinske literature, poleg tega pa je pri tem razbrati poseben odnos do maternega jezika nasploh. Avtorji, ki se odločijo za pisanje v narečju, svojo izbiro izpostavljajo tako, da se tudi delno oddaljujejo od ostalih piscev, ker zelo po-

29 Zudič Antonič, Storia e antologia della letteratura italiana di Capodistria, Isola e Pirano, 393. 
udarjajo svoje korenine v kraju, kjer so bili rojeni in kjer so živeli.

Ključne besede: manjšinska književnost, narečna književnost, Slovenci v Italiji, Italijani v Sloveniji in na Hrvaškem, odnos do jezika

\section{Summary}

The purpose of this paper is to demonstrate that the dialectal literature is one of the typical expression of the minority literature. Previous studies have shown that various dialect literatures arise first on the outskirts of an ethnic settlement area, in neighbouring countries and even in those areas where the language comes into contact with other languages and cultures. In the same areas also the relationship with the mother tongue and with the dialect itself is much more distinguished and significant. Analysing the cases of two minority communities, the Slovenian in Italy and the Italian minority in Slovenia and Croatia, the author tries to prove that the dialect literature is a very typical phenomenon in minority literature, in which it can be noticed a special relationship to language in general. The authors that decide to write in dialect, do it with special purposes trying to distinguish themselves from the others and to emphasise their linguistic and cultural roots in the land, where they were born and where they live.

Keywords: minority literature, dialect literature, Slovenians in Italy, Italians in Slovenia and Croatia, relationship to the language

\section{Bibliografia}

Bandelj, David. Rod Lepe Vide: antologija sodobne poezije Slovencev v Italiji. Ljubljana: Študentske založba, 2009.

Bandelj, David, "Literature of Slovenians in Italy: A subsystem of Slovenian supernational system? " Interlitteraria 5 , no. 2 (2010): 432-44I.

Bartol, Vladimir. "Predgovor." In Souze jn smeh, ur. Marija Mijot, 7-9. Trst: Založništvo tržaškega tiska, 1968.

Berruto, Gaetano. Introduzione all'italiano contemporaneo, La variazione e gli usi. Roma-Bari: Laterza, 2003.
Brevini, Franco. Le parole perdute. Dialettie poesia del nostro secolo. Torino: Einaudi, 1990.

Cergol, Jadranka. "Some Typological Features of ,Minority' Literatures: the Case of the Slovenian and Italian Minorities." L'analisi linguistica e letteraria 24, no. I (2016): 6I76.

Cesaretti Salvi, Luigi Maria, "La letteratura dialettale.” Enciclopedia Treccani (2006), http://www.treccani.it/enciclopedia/ letteratura-dialettale_\%28EnciclopediaItaliana\%29/ (28-4-2017)

Contini, Gianfranco. La letteratura italiana. Firenze-Milano: Sanoni-Accademia, 1974. Curto, Sergio. Meingule insanbrade = Briciole sparse. Trieste : Unione degli italiani dell'Istria e di Fiume: Università popolare, 1983.

Deghenghi Olujić, Elis, Miran Košuta. Drugačni verzi / Versi diversi. KoperCapodistria: Unione italiana, 2006. 1

( ( $\checkmark$

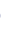
$\sqrt{2-10}$ Hladnik, Miran. "Regionalism in Slovene Rural Prose." Slovene Studies I3, no. 2 (I99I): I 43-I53.

Hočevar, Jože. "Pride vse en vse pasá." In: Pride vse en vse pasá (Pesmi o Istri in njenih ljudeb), ur. Nelda Štok-Vojska, I 43-I 48. Marezige: samozaložba, 1999.

Košuta, Miran. E-maili: eseji o mejni literaturi. Maribor: Litera, 2008.

Marabini, Claudio. "Poesia contemporanea in lingua e dialetto: quale rapporto?” In $\mathrm{La}$ maschera del dialetto, ed. Andrea Foschi, Elio Pezzi, 64-72. Ravenna: Longo, 1988.

Milani, Nelida, e Roberto Dobran, Le parole rimaste. Pula: Pietas Iulia; Rijeka: Edit, 2010.

Mijot, Marija. Souze jn smeh. Trst: Založništvo tržaškega tiska, 1962.

Novak Popov, Irena. "Razmišljanje o sodobni narečni poeziji.” In Slovenska narečja med sistemom in rabo, ur. Vera Smole, 435-453. Ljubljana: Znanstvena založba Filozofske fakultete, 2009. 
Pirjevec, Marija. "Periodizacija slovenske književnosti na Tržaškem (od I6. do 20. stoletja)." Annales, Series historia et sociologia 21, no. 2 (2011): 353-362.

Pirjevec, Marija, Questa Trieste. Trieste:

Mladika, 2016.

Sosič, Marko. Tito, amor mijo. Trieste:

Comunicarte, 2012.

Stanonik, Marija. Slovenska narečna

književnost. Maribor: Slavistično društvo Maribor, 2007.

Zanini, Ligio. Martin Muma. Fiume: Edit, 1999.

Zudič Antonič, Nives. Storia e antologia della letteratura italiana di Capodistria, Isola e Pirano. Capodistria: Unione italiana, 2014.

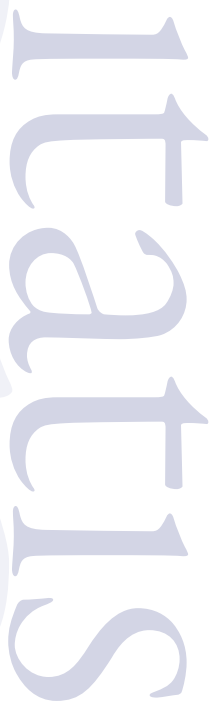

